\title{
Avaliação psicológica e ética: um estudo com universitários
}

\author{
Adauto Garcia Jr. ${ }^{1}$ \\ Cássia Aparecida Bighetti ${ }^{2}$ \\ Fernanda Andrade de Freitas ${ }^{3}$ \\ Yeda Cícera Oswaldo ${ }^{4}$ \\ Ana Paula Porto Noronha ${ }^{5}$
}

\section{Resumo}

A ética é considerada um fator importante nas avaliações psicológicas e na atuação do psicólogo, como em qualquer área de atividade profissional. O presente estudo objetivou compreender os fatores éticos no processo de avaliação psicológica. Foram participantes desse estudo 126 alunos de $1^{\circ}, 2^{\circ}, 3^{\circ}, 4^{\circ}$, $5^{\circ}, 9^{\circ}$ e $10^{\circ}$ semestres do curso de psicologia de uma universidade particular do interior do estado de São Paulo. Desse total, a maioria era do sexo feminino. A média de idade foi de 25,69. O instrumento utilizado continha 24 itens com três possibilidades de respostas dispostas em escala likert.A coleta de dados ocorreu em uma única sessão, coletivamente, em sala de aula. Esse estudo possibilitou discutir que o tempo de formação profissional influencia na forma como os alunos conciliam ética nas situações práticas que envolvam a avaliação psicológica.

Palavras-chave: Prática profissional. Estudantes de psicologia. Código de ética.

\footnotetext{
${ }^{1}$ Mestre em Psicologia pela Universidade São Francisco e docente do Centro Universitário Adventista de São Paulo - UNASP.

${ }^{2}$ Doutoranda em Psicologia da Universidade São Francisco. Mestre em Psicologia Escolar pela PUCCAMP, docente da Universidade São Francisco.

${ }^{3}$ Doutoranda em Educação pela UNICAMP e docente da Universidade São Francisco.

${ }^{4}$ Doutoranda em Psicologia da Universidade São Francisco. Mestre em Educação pela Universidade Metodista de Piracicaba. Diretora Acadêmica e Docente do curso de Psicologia das Faculdades Integradas Einstein de Limeira.

${ }^{5}$ Doutora em Psicologia: Ciência e Profissão pela PUC-Campinas. Docente do Programa de Pósgraduação em Psicologia da Universidade São Francisco.
} 


\section{Introdução}

A avaliação psicológica configura-se como importante atividade do psicólogo, em qualquer que seja o campo de atuação profissional. Tem sido definida como um processo de análise e interpretação de dados relativos às amostras de comportamento e traços psicológicos (AERA - American Educational Research Association, APA - American Psychology Association \& NCME - National Council on Measurement in Education, 1999). A avaliação psicológica utiliza uma variedade de técnicas e procedimentos que permitem coletar tais dados, dentre os quais se encontra a aplicação de testes psicológicos.

No Brasil, os testes psicológicos são instrumentos de uso privativo dos psicólogos, com base na Lei $\mathrm{n}^{\circ}$ 4.119/62 (CFP, 2001). Podem ser utilizados em vários contextos de atuação, tais como escolas, empresas, avaliação de trânsito e clínicas-escolas de universidades, dentre outros, com fins diagnósticos, interventivos ou de pesquisa.

É notório o fato de que cada vez mais áreas e contextos profissionais requerem a utilização da testagem psicológica, despertando interesse maior. Entretanto, à medida que a testagem psicológica expande suas áreas de aplicação, o risco de mau uso dos instrumentos e faltas éticas variadas tende a aumentar.

À preocupação com a evitação de problemas que comprometem a avaliação psicológica, relacionados ao mau uso de testes e faltas éticas, deve estar inclusa a devida atenção à formação acadêmica do profissional. No sentido desta preocupação, $\mathrm{o}$ presente estudo se dispôs a investigar a visão e o grau de compreensão de estudantes de psicologia, em diferentes etapas do curso, sobre diversas questões éticas relacionadas à atuação em avaliação psicológica.

\section{Avaliação psicológica e ética profissional}

Estudos como o realizado por Pasquali e Alchieri (2001), traçaram um delineamento histórico de tal modo que apontam o ano de 1907 como o marco da fundação do primeiro laboratório de psicologia no Brasil, no Hospital Nacional de Alienados, sendo que a utilização do teste de inteligência de Binet por médicos do hospital se deu a partir de 1913. Desde então, pode-se notar interesse crescente, refletido no número de pesquisas, na área da avaliação psicológica. 
As últimas décadas foram marcadas por críticas ao uso de testes na psicologia, conforme aponta Wechsler (1999), o que desestimulou a pesquisa, promovendo considerável declínio no avanço científico da área. Apesar disso, ao longo dos últimos anos, os testes psicológicos retomaram sua consideração e importância, tanto em âmbitos nacionais quanto internacionais e sua., relevância profissional não precisa mais ser questionada, conforme ressaltam Noronha e Alchieri (2002) e Noronha (2006). O aumento das publicações internacionais, relacionadas aos instrumentos psicológicos, pode ser verificado a partir da década de 80 , conforme Anastasi e Urbina (2000), como indício do retomado interesse pelos instrumentos de avaliação psicológica.

Fatores que desencadeiam resistência e crítica ao uso de testes estão relacionados, em parte, à qualidade questionável e/ou mau uso desses por parte de alguns profissionais. Nessa direção, o estudo realizado por Noronha e Alchieri (2002) apontou sérios problemas relacionados à construção e utilização de testes psicológicos, que se referem à ausência de pesquisas que investigam os parâmetros psicométricos e à má qualidade de. instrumentos comercializados. Tais aspectos não favorecem uma avaliação psicológica adequada e científica.

A partir do que Pasquali (1999) destaca, um dos motivos que comprometem a qualidade dos testes pode ser devido ao fato de que, durante muitos anos, os psicólogos brasileiros acomodaram-se no uso de testes estrangeiros meramente traduzidos, sem se preocuparem em verificar a qualidade dos mesmos e padronização para a população nacional.

Outra questão a ser considerada refere-se à importância da ética vinculada à utilização dos instrumentos psicológicos. Para Chauí (2002) a ética pode ser um conjunto de regras, princípios ou maneiras de pensar que guiam ações de um grupo particular (moralidade), ou o estudo sistemático da argumentação sobre como devemos agir. Sob essa perspectiva, ética é uma palavra grega, com dois significados possíveis. O primeiro está relacionado à palavra éthos, escrita com vogais longas, que pode ser traduzida por "costume" ou escrita com vogais breves, que significa propriedade do caráter. A primeira serviu de base para a tradução latina "moral", enquanto a segunda, de alguma forma, orienta a utilização atual que damos à palavra "ética" (JARDILINO, 1998). 
Para Vazque (1994), a palavra ética significa uma teoria ou ciência do comportamento moral dos homens em sociedade; já Valls (1998) a define como estudo das ações ou dos costumes, e pode ser a própria realização de um tipo de comportamento, compreendida como as disposições do homem na vida, o seu caráter, costumes e a própria moral.

No contexto moral e social, a ética se responsabiliza pelos questionamentos, pela responsabilidade ético-social e pela ação como profissionais numa sociedade marcada por tradições arcaicas e com moralidade individualizada. Lamentavelmente, de acordo com Jardilino (1998), os códigos de ética profissionais constituem-se de regras e normas muitas vezes fora do contexto atual. $\mathrm{O}$ autor afirma, no que se refere à elaboração de um código de ética profissional, que ela deve objetivar a leitura da sociedade de forma que a conduta seja monitorada pelos valores morais, que por sua vez, se relacionam com o perfil daquele profissional no contexto social no qual está inserido.

Ainda nesse sentido, para o Conselho Federal de Psicologia (1987) "O código de ética é a expressão da identidade profissional daqueles que nele vão buscar inspirações, conselhos e normas de conduta. Ele é, ao mesmo tempo, uma pergunta e uma resposta. É um apelo-pergunta no sentido de se ver o ser humano não apenas como uma unidade isolada, mas como um subsistema de um grande sistema" (p.Ol).

A ética precisa resguardar os interesses dos indivíduos no seio de sua coletividade por meio de regras pelas quais todos deverão ser responsáveis pelos danos causados a terceiros. Assim, o código de ética dos psicólogos tenta resguardar os direitos das pessoas, à medida que confere condições para a atuação profissional. Ao abordar-se a relação entre avaliação psicológica e ética, constata-se que a formação profissional torna-se um espaço importante nesse sentido, pois é um contexto em que essas práticas são discutidas e integradas.

Em estudo realizado por Noronha (2002), estão descritas três categorias de problemas quanto ao uso dos testes psicológicos, na visão de 214 profissionais, a saber, os relacionados à deficiência do próprio instrumento, à utilização desses instrumentos e a formação profissional. Segundo Anastasi e Urbina (2000) a formação profissional deve ser eficiente e garantir preparação adequada aos futuros 
psicólogos, tomando-os competentes, dentre outras funções, para a utilização adequada de instrumentos de avaliação psicológica. Nesse sentido, o Fórum de Discussão promovido pelo Conselho Federal de Psicologia, apontou, também, problemas relacionados à formação profissional em avaliação psicológica como a deficiência e carência de fundamentação teórica e conhecimento adequado dos testes (CFP, 1997).

Ainda no que se refere à formação profissional, destaque deve ser dado à ética na utilização e aplicação dos testes psicológicos. Essa preocupação foi apontada por Alves, Rosa e Pellini (2005) que retratam a realidade das universidades brasileiras, nas quais os professores aplicam os testes psicológicos nos próprios alunos, disponibilizando seus resultados por meio do acervo de bibliotecas para toda a população. Alunos, em trabalhos de conclusão de curso, aplicam testes psicológicos sem avaliarem adequadamente as condições éticas. Tal prática evidencia a necessidade de agregar questões éticas à formação, envolvendo as disciplinas de avaliação psicológica.

A inserção da questão ética se faz necessária para fornecer subsídios aos alunos, futuros profissionais, no que concerne às responsabilidades para a utilização dos testes, de tal sorte que a preparação permita uma atuação ética e profissional. Para o ITC (2003), é necessário compreender que a ética considera alguns componentes como o uso competente dos testes, assumir responsabilidades no uso dos mesmos, a segurança no arquivamento dos dados obtidos e assegurar a confidencialidade dos resultados, para que, na prática, esses alunos consigam avaliar o potencial dos testes em uma situação de avaliação qualquer. Além disso, é importante que os alunos estejam preparados para selecionar adequadamente testes tecnicamente confiáveis em cada situação, prestar devida consideração às condições culturais, preparar cuidadosamente as sessões de aplicação, analisar os resultados adequadamente, apresentando-os de forma clara para as pessoas envolvidas.

Considerando as questões abordadas, relacionadas às condições de formação e ética no contexto da avaliação psicológica, o presente trabalho procurou analisar como estudantes de psicologia, em diferentes momentos de formação e pautados em seu conhecimento sobre ética profissional, julgam a atuação do psicólogo no contexto da avaliação psicológica. 


\section{Método}

\subsection{Participantes}

Participaram da presente pesquisa 126 estudantes do Curso de Psico$\operatorname{logia} \operatorname{dos} 1^{\circ}, 2^{\circ}, 3^{\circ}, 4^{\circ}, 5^{\circ}, 9^{\circ}$ e $10^{\circ}$ semestres de uma Universidade particular do interior de São Paulo. Desse total 76,2\% (N=96) eram do sexo feminino e $22,2 \%(\mathrm{~N}=28)$, do sexo masculino. A idade dos sujeitos variou entre 17 a 52 anos, com a media de 25,69 (DP=8,65).

\subsection{Material}

O instrumento continha duas partes, sendo a primeira dedicada a identificação dos participantes, ou seja, informações quanto a sexo, idade e semestre de formação que o o sujeito se encontra.

A segunda parte, constituiu-se no questionário fechado composto de 24 afirmativas relacionadas à visão do estudante sobre a ética na prática em avaliação psicológica. Os itens são: 1) Fazer algumas pequenas modificações em um determinado instrumento, a fim de facilitar a aplicação no cliente; 2) Emitir um parecer sobre um cliente e não ser fiel aos dados coletados; 3) Adulterar os resultados de uma avaliação psicológica; 4) Ajudar o cliente a responder as questões de um teste que tinha como objetivo avaliar a inteligência; 5) Apresentar ao juiz informações de fatos que não eram relevantes ao processo judicial; 6) Disponibilizar os resultados dos testes psicológicos do cliente via on line; 7) Utilizar determinados testes psicológicos não recomendados pelo conselho Federal de Psicologia CFP; 8) Numa sessão de aplicação de testes, interromper o processo de avaliação para atender outros clientes; 9) O assistente do psicólogo aplicar testes psicológicos no cliente, o processo de avaliação, para obtenção de carteira de motorista; 10) Identificar nome, idade, endereço dos sujeitos que participaram de uma pesquisa de avaliação psicológica; 11) Criar um instrumento para avaliar a inteligência com base na sua experiência clínica e aplicar em alguns pacientes; 12) Ao fazer uma avaliação psicológica e perceber que tem apenas uma folha de resposta do teste a ser aplicado, o psicólogo recorre a uma papelaria para reproduzir mais cópias; 13) Emprestar um instrumento de avaliação psicológica a um colega de profissão; 14) Aplicar um instrumento de habilidades sociais no próprio 
filho; 15) Trabalhar na área organizacional e não ter o registro no CRP; 16) Atuar em uma instituição e não utilizar somente testes padronizados, mas recorrer, quando necessário, a testes publicados em revistas semanais; 17) Fornecer informações confidenciais da avaliação psicológica realizada em candidatos para a chefia da empresa solicitante; 18) Em um anuncio de jornal, oferecer um pacote promocional de sessões de avaliação psicológica; 19) Informar à criança os resultados obtidos nos testes durante o psicodiagnóstico; 20) Revelar informações sigilosas à família de um cliente potencialmente suicida; 21) Elaborar um laudo com base apenas em algumas entrevistas; 22) Decidir avaliar por meio de uma bateria de testes psicológicos, um parente que parece estar depressivo; 23) Utilizar, no processo de seleção de candidatos para uma determinada empresa, dentre os instrumentos de avaliação psicológica, a grafologia; 24) Emitir a devolutiva do desempenho a um candidato à carteira de habilitação que realizou três testes psicológicos.

Os itens foram avaliados em escala likert de 0 a 3 , sendo que o 0 indicava não houve falta ética, o 1 indicava houve falta ética leve ou moderada, o 2 houve falta ética grave e o 3 não sei avaliar.

\subsection{Procedimento}

Para a realização da coleta de dados, foi pedido permissão aos docentes responsáveis pelas salas de aulas. Com o consentimento, foi explicado aos alunos os objetivos do estudo e na seqüencia o convite para o preenchimento do instrumento. A coleta de dados foi coletiva, em uma única sessão com a duração de aproximadamente trinta minutos, e obedeceu as diretrizes estabelecidas para a realização de pesquisas científicas com seres humanos.

\subsection{Plano de análise de dados}

Para a tabulação dos dados foi utilizada a planilha eletrônica denominada SPSS (Statistical Package for the Social Sciences). As respostas dos alunos foram ordenadas de acordo com a escala likert atribuída por cada um. Deste modo, os dados puderam ser submetidos à análise descritiva, consistência interna e a diferença de médias. 


\section{Resultados}

\subsection{Estatística descritiva}

Foi realizada a análise descritiva dos resultados, cujos dados são apresentados a seguir. A média de acerto da amostra foi 29,83 e o $\mathrm{DP}=4,68$, sendo que as pontuações variaram de 16 a 38 de um total de 48 pontos possíveis. A análise dos itens indicou que os itens 2, 3 e 16 apresentaram os maiores índices de acerto, 96,0\%, 99,2\% e 82,5\% respectivamente, enquanto os itens 13 e 23 obtiveram os menores índices de acerto, $24,6 \%$ e $22,2 \%$.

Em contrapartida, os itens 12, 14 e 20 apresentaram-se mais distribuídos entre duas ou três possibilidades de respostas, apontando indícios de maior dificuldade, visto que os participantes se dividiram dentre as respostas possíveis. O item 20 chamou a atenção especialmente pelo fato de que os sujeitos se dividiram principalmente entre duas respostas extremas, ou seja, enquanto $50 \%(\mathrm{~N}=63)$ da amostra optou por responder que a proposição não representava falta ética, 39,7\% (N=50) respondeu que envolve falta ética grave.

Ainda nesse sentido, ou seja, o da análise das freqüências das respostas, o item 21 destacou-se por dividir as respostas dos sujeitos de forma equilibrada entre as opções 0 (não houve falta ética) e 1 (houve falta ética leve/moderada) dentre as outras possibilidades 2 (houve falta ética grave) e 3 (não sei avaliar) o que indica uma razoável dificuldade de interpretar se houve ou não falta ética. As freqüências por item podem ser visualizadas na Tabela 1, e as opções consideradas corretas aparecem em negrito.

\subsection{Precisão}

As diferentes formas para se obter a precisão dos instrumentos, são: teste-reteste, formas alternadas e as análises de consistência interna, que pode ser obtida pela forma Kuder-Richardson e Alfa de Cronbach. Lembrando que, a variação refletida nos resultados dos examinandos, obtidos por meio da aplicação dos testes e as suas causas são gerais e não relacionadas com os objetivos da avaliação (APA; AERA; NCME, 1999). Essa variação pode ser informada por meio da variância, desvio-padrão, erro padrão de medida e a Teoria de Resposta ao Item (TRI) e pode ser, então, compreendida como a precisão. Para o instrumento que os autores elaboraram, a consistência interna foi estabelecida por meio do Alfa de Cronbach. 
Univ. Ci. Saúde, Brasilia, v. 5, n. 1/2, p. 61-74, 2007

Avaliação psicológica e ética: um estudo com universitários

Tabela 1 - Freqüência das respostas dos sujeitos

\begin{tabular}{|c|c|c|c|c|c|c|}
\hline & \multicolumn{2}{|c|}{0} & \multicolumn{2}{|c|}{1} & \multicolumn{2}{|c|}{2} \\
\hline ITEM & $\mathrm{F}$ & $\%$ & $\mathrm{~F}$ & $\%$ & $\mathrm{~F}$ & $\%$ \\
\hline 1 & 25 & 19,8 & 39 & 31,0 & 61 & 48,4 \\
\hline 2 & - & - & 5 & 4,0 & 121 & 96,0 \\
\hline 3 & - & - & 1 & 0,8 & 125 & 99,2 \\
\hline 4 & 1 & 0,8 & 100 & 79,4 & 24 & 19,0 \\
\hline 5 & 27 & 21,4 & 65 & 51,6 & 33 & 26,2 \\
\hline 6 & 15 & 11,9 & 14 & 11,1 & 96 & 76,2 \\
\hline 7 & 5 & 4,0 & 21 & 16,7 & 100 & 79,4 \\
\hline 8 & 5 & 4,0 & 78 & 61,9 & 43 & 34,1 \\
\hline 9 & 19 & 15,1 & 32 & 25,4 & 75 & 59,5 \\
\hline 10 & 37 & 29,4 & 26 & 20,6 & 63 & 50,0 \\
\hline 11 & 41 & 32,5 & 19 & 15,1 & 66 & 52,4 \\
\hline 12 & 38 & 30,2 & 40 & 31,7 & 48 & 38,1 \\
\hline 13 & 31 & 24,6 & 23 & 18,3 & 71 & 56,3 \\
\hline 14 & 40 & 31,7 & 35 & 27,8 & 51 & 40,5 \\
\hline 15 & 23 & 18,3 & 18 & 14,3 & 83 & 65,9 \\
\hline 16 & 6 & 4,8 & 16 & 12,7 & 104 & 82,5 \\
\hline 17 & 93 & 73,8 & 14 & 11,1 & 18 & 14,3 \\
\hline 18 & 25 & 19,8 & 24 & 19,0 & 75 & 59,5 \\
\hline 19 & 30 & 23,8 & 26 & 20,6 & 68 & 54,0 \\
\hline 20 & 63 & 50,0 & 12 & 9,5 & 50 & 39,7 \\
\hline 21 & 71 & 56,3 & 50 & 39,7 & 4 & 3,2 \\
\hline 22 & 20 & 15,9 & 40 & 31,7 & 65 & 51,6 \\
\hline 23 & 78 & 61,9 & 19 & 15,1 & 28 & 22,2 \\
\hline 24 & 65 & 51,6 & 20 & 15,9 & 39 & 31,0 \\
\hline
\end{tabular}

Foram obtidos três coeficientes de consistência de acordo com os diferentes momentos da formação desses alunos, subdivididos da seguinte forma: $1^{\circ} \mathrm{e} 2^{\circ}$ semestres - Grupo 1 composto por $\mathrm{N}=34$ sujeitos; $3^{\circ}, 4^{\circ}$ e $5^{\circ}$ semestres - Grupo 2 composto por $\mathrm{N}=70$ sujeitos; e $9^{\circ}$ e $10^{\circ}$ semestres - Grupo 3 com- 
posto por $\mathrm{N}=18$ sujeitos. O primeiro grupo obteve um $\alpha=0,46$, o segundo grupo obteve um $\alpha=0,44$, já o terceiro obteve $\alpha=0,5$. A consistência obtida para o instrumento englobando os diferentes grupos é de $\alpha=0,43$. Observouse que os alunos do último ano apresentaram maior consistência quanto ao instrumento demonstrando ser um grupo, que pela proximidade do término da graduação, tem maior possibilidade de julgar as situações práticas do psicólogo em relação aos outros grupos.

\subsection{Análise de variância}

Os sujeitos pesquisados foram divididos em três grupos no que se refere ao semestre letivo. Sendo assim a ANOVA não apresentou diferença significativa na forma como os grupos responderam ao instrumento $F(3,126=0,653$; $\mathrm{p}=0,862$ ). Os dados indicaram que contrariamente ao que se esperava a média do desempenho não aumentou conforme o aumento do semestre. Dessa forma, o grupo de alunos do último ano apresentou desempenho inferior ao grupo intermediário e do primeiro ano. Essa constatação não corrobora a hipótese de que o conhecimento agregado pelo curso, principalmente os conteúdos relacionados à questão da avaliação psicológica e ética profissional, permitiram um domínio maior da área que se refletiu em melhor desempenho, diretamente relacionado ao tempo de curso e a quantidade de informações recebidas.

Entretanto, deve ser levado em consideração que o grupo intermediário é composto por sujeitos que cursam o terceiro, quarto e quinto semestre do curso de psicologia, que, segundo a grade da universidade pesquisada, são os semestres que os alunos têm contato com as disciplinas diretamente relacionadas com a avaliação psicológica. Esse dado pode indicar uma influência maior do aprendizado do conteúdo dessas disciplinas nas respostas dos sujeitos, indicando uma possível razão para o grupo intermediário ter apresentado média de desempenho acima dos demais. Esse mesmo dado, porém, não explica o fato do grupo do primeiro ano ter apresentado desempenho mais próximo ao do grupo intermediário que o grupo do último ano. As médias de desempenho de acordo com o semestre letivo podem ser visualizadas na Tabela 2 . 
Tabela 2 - Desempenho por grupo de semestre

\begin{tabular}{cccc}
\hline & Média & DP & N \\
\hline Grupo 1 (sem 1 e 2) & 29,79 & 4,97 & 34 \\
Grupo 2 (sem 3, 4 e 5) & 30,37 & 4,47 & 70 \\
Grupo 3 (sem 9 e 10) & 28,38 & 4,94 & 18 \\
\hline
\end{tabular}

\section{Discussão}

Esse trabalho apresentou uma avaliação do julgamento que os alunos de Psicologia fazem quanto à atuação do psicólogo no contexto da Avaliação Psicológica. Os dados nos permitem refletir a respeito da formação acadêmica dos alunos e o seu julgamento ético voltado para uma área específica da Psicologia.

A análise descritiva dos itens demonstra que a maioria dos alunos acertou a maior parte dos itens, indicando que o instrumento foi fácil para essa amostra. Embora, alguns deles tenham apresentado uma distribuição proporcional em todas as possibilidades de respostas do instrumento, indicando assim que há itens muito difíceis dos alunos julgarem como ocorrendo ou não falta ética, a saber, "ao fazer uma avaliação psicológica e perceber que tem apenas uma folha de resposta do teste a ser aplicado, o psicólogo recorre a uma papelaria para reproduzir mais cópias", "aplicar um instrumento de habilidades sociais no próprio filho", "revelar informações sigilosas à família de um cliente potencialmente suicida" e "elaborar um laudo com base em apenas algumas entrevistas".

A dificuldade de julgamento em relação a alguns itens demonstra que os alunos em formação necessitam cada vez mais dos conhecimentos acadêmicos relacionados à vivência que são proporcionadas na prática, sempre considerando as questões éticas e a avaliação psicológica. Esse dado evidencia a pouca apropriação do código de ética da profissão por parte dos alunos, pois as situações práticas descritas no instrumento podem ser interpretadas e julgadas à luz dos parágrafos contemplados no Manual de Conduta do Psicólogo (1999).

Atualmente, presenciam-se muitos profissionais que são penalizados, por não se pautarem pelo código de ética em sua atuação (Freitas, 2005), talvez pela falta do conhecimento necessário ou até mesmo por não seguir um código de conduta. Segundo Anastasi e Urbina (2000) a formação profissional deve ser eficien- 
te garantindo uma preparação adequada aos futuros psicólogos, principalmente quanto à utilização dos instrumentos psicológicos.

Em função disso e com base nas análises de precisão, é possível afirmar que os alunos do último ano apresentaram maior capacidade de julgamento ao relacionar o que é ético e não ético frente às situações práticas de avaliação psicológica. Isso pode ser justificado, entre outros motivos, pela questão do tempo de formação que eles se encontram o que possibilita uma visão mais apurada das situações práticas do Psicólogo e a sua relação com os conteúdos teóricos assimilados durante o processo acadêmico.

\section{Conclusões}

Esse estudo possibilitou discutir que o tempo de formação profissional influencia na forma como os alunos conciliam ética nas situações práticas que envolvam a avaliação psicológica. Ao lado disso, permite uma reflexão a respeito de uma questão acadêmica fundamental, qual seja, a importância da aproximação dos conteúdos acadêmicos à prática profissional.

Os resultados indicam que o desempenho dos estudantes em relação ao instrumento utilizado não confirmou claramente a hipótese inicial de que os alunos deveriam apresentar melhor desempenho quanto ao conhecimento de questões éticas da avaliação psicológica na medida em que se atingem etapas mais adiantadas do curso. Por outro lado, permitiu o levantamento da hipótese de que os alunos que estão nas etapas do curso em que se ministram as disciplinas relacionadas à avaliação psicológica, apresentaram melhor desempenho, fato que pode acentuar a influência da aprendizagem acadêmica para a formação de conceitos.

Entretanto, esse estudo não teve a pretensão de esgotar esses assuntos sendo necessária a realização de mais pesquisas para a constatação de dados mais significativos acerca do tema. Algumas sugestões para estudos posteriores podem ser em torno do aprimoramento do instrumento para a coleta de dados, tendo em vista alguns dos seus itens descritos como "fáceis" e outros como "difíceis", o que possivelmente dificultou a compreensão do fenômeno a pesquisar. 


\section{Psychological assessment and ethics: a study with university students}

\section{Abstract}

Ethics is considered an important factor in psychological analyses and in the psychologist practice, as any professional activity. This present study aimed to understand the ethical factors in the psychological assessment process. One hundred twenty-six of first, second, third, fourth, fifth, ninth and tenth Psychology graders of a private university in the country side of Sao Paulo had participated in this study. Most of them were women (age average $=25.69$ ). The instrument was formed by 24 tripled-answer items disposed on a Likert scale. Data was collectively collected in the classroom in a single section. This study has shown the possibility to discuss that professional formation time influences the way how the students mediate ethic and practical situations which involve psychological assessment.

Keywords: Professional practice. Psychology students. Ethics code.

\section{Referências}

AMERICAN EDUCATIONAL RESEARCH ASSOCIATION; AMERICAN PSYCHOLOGY ASSOCIATION; NATIONAL COUNCIL ON MEASUREMENT IN EDUCATION. Standards for educational and psychological testing. New York: American Educational Research, 1999.

ALVES, J. C. B. et al. Aspectos éticos e práticos relativos a guarda e consulta de testes psicológicos nas universidades. In: CONGRESSO DE AVALIAÇÃO PSICOLÓGICA, 2, 2005, Gramado, Anais. Gramado: [s.n.], 2005.

ANASTASI, A.; URBINA, S. Testagem psicológica. Porto Alegre: Artes Médicas, 2000.

CHAUÍ, M. Convite à filosofia, São Paulo: Ática, 2002.

CONSELHO FEDERAL DE PSICOLOGIA. Resolução n. ${ }^{\circ}$ 010/05. Código de Ética Profissional do Psicólogo, Brasília, 2005.

CONSELHOFEDERALDEPSICOLOGIA. Resoluçãon. 25/2001. Disponível em:<www. pol.org.br/pesquisa/resposta_pesquisa.cfm?_area=15>. Acesso em: 04/10/2002. 
CONSELHO FEDERAL DE PSICOLOGIA. Resolução n. ${ }^{\circ}$ 0021/1987. Código de ética profissional do psicólogo. Brasília, 1987.

CONSELHO REGIONAL DE PSICOLOGIA. Manual do Conselho Regional de Psicologia. São Paulo: CRP-06, 1999.

FREITAS, F. A. Processos éticos: principais artigos infringidos pelos psicólogos. In: ENCONTRO NACIONAL DA ABEP: FORMAÇÃO EM TRANSFORMAÇÃO EM FORMAÇÃO, Perdizes, SP. Resumos. Perdizes, SP: Associação Brasileira de Ensino de Psicologia, p. 32, 2005.

INTERNATIONAL TEST COMISSION. Diretrizes para o uso dos testes: International Test Comission. Tradução realizada pelo Instituto Brasileiro de Avaliação Psicológica .2003.

JARDILINO, J. R. L. Ética: subsídios para formação de profissionais da área da saúde. São Paulo: Pancaft, 1998.

PASQUALI, L. Testes Psicológicos: conceitos, história, tipos e usos. In: PASQUALI. L. (Org.). Técnicas de exame psicológico. São Paulo: Casa do Psicólogo, 2001. p. 13-56.

PASQUALI, L. Instrumentos psicológicos: manual prático de elaboração. Brasília: LabPAM / IBAPP, 1999.

PASQUALI, L.; ALCHIERI, J. C. Os testes psicológicos no Brasil. In: PASQUALI, L (Org.). Técnicas de exame psicológico. São Paulo: Casa do Psicólogo, 2001. p. 195221.

NORONHA, A. P. P. Os problemas mais graves e mais freqüentes no uso dos testes psicológicos. Psicologia: reflexão e crítica, [S.l.], v.15, p. 135-142, 2002.

NORONHA, A. P. P. Os testes psicológicos comercializados no Brasil. Psychologica, [S.1.], v. 43, p. 227-244, 2006.

NORONHA, A. P. P. ; ALCHIERI, J. C. Reflexões sobre os instrumentos de avaliação psicológica. In: PRIMI, R. (Org.). Temas em avaliação psicológica. Campinas: Impressão Digital do Brasil, 2002. p. 7-16.

WECHSLER, S. M. Guia de procedimentos éticos para a avaliação psicológica. In: WECHSLER, S. M.; GUZZO, R. S. L. (Org.). Avaliação psicológica: perspectiva internacional.. São Paulo: Casa do Psicólogo, 1999. p.133-141. 